\title{
Strong topical steroid, NSAID, and carbonic anhydrase inhibitor cocktail for treatment of cystoid macular edema
}

This article was published in the following Dove Press journal:

International Medical Case Reports Journal

I December 2015

Number of times this article has been viewed

\section{Masumi G Asahi \\ Gabriela L Bobarnac \\ Dogaru \\ Spencer M Onishi \\ Ron P Gallemore}

Retina Macula Institute, Torrance, CA, USA
Correspondence: Ron P Gallemore Retina Macula Institute, 420I Torrance Boulevard Ste 220, Torrance, CA 90503, USA

Tel + I 3109449393

Fax + I 3109443393

Email rongallemoremd@gmail.com
Purpose: To report the combination cocktail of strong steroid, non-steroidal anti-inflammatory drug (NSAID), and carbonic anhydrase inhibitor drops for treatment of cystoid macular edema.

Methods: This is a retrospective case series of patients with cystoid macular edema managed with a topical combination of strong steroid (difluprednate), NSAID, and carbonic anhydrase inhibitor drops. The patients were followed with optical coherence tomography and fluorescein angiography.

Results: In our six cases, resolution of the cystic edema with improvement in visual acuity was achieved with the use of a combination cocktail of drops. Leakage on fluorescein angiography and cystic edema on optical coherence tomography both responded to treatment with the topical cocktail of drops.

Conclusion: A topical cocktail of strong steroid, NSAID, and carbonic anhydrase inhibitor drops are effective for managing cystoid macular edema. Further studies comparing this combination with more invasive treatments should be undertaken to determine the efficacy of this cocktail over other treatment options.

Keywords: birdshot chorioretinopathy, diabetic macular edema, retinal vein occlusion

\section{Introduction}

Cystoid macular edema (CME) is associated with a variety of ocular conditions, including postoperative inflammation, retinal vein occlusions, uveitis, and diabetic retinopathy. It continues to be a blinding disorder and indeed is also termed cystoid macular degeneration, since chronic CME leads to macular atrophy and vision loss to the 20/200 level. ${ }^{1}$ Even mild cases can erode vision line-by-line over many years, so early diagnosis and aggressive treatment is often warranted.

First-line therapy for CME is topical anti-inflammatory drops, namely, steroids and non-steroidal anti-inflammatory drugs (NSAID) with greater gains of visual acuity, faster recovery, and greater likelihood of improvement in contrast sensitivity compared to monotherapy with either agent alone. ${ }^{2}$ When $\mathrm{CME}$ is recalcitrant to topical drops alone, periocular and intravitreal steroid injections may be effective. ${ }^{3}$ Furthermore, in select cases, oral acetazolamide, a carbonic anhydrase inhibitor (CAI), has been effective in the management of $\mathrm{CME}^{4}$

Here we report a combination cocktail of strong steroid (difluprednate $0.05 \%$ ), NSAID, and carbonic anhydrase inhibitor (CAI) drops for the treatment of CME associated with a variety of conditions, including recalcitrant cases previously treated with other methods. Difluprednate $0.05 \%$ is regarded as a strong steroid as it has been reported to be 56 times more effective at binding glucocorticoid receptors than the $1 \%$ formulation of prednisolone acetate which is routinely used for 
the treatment of CME and ocular inflammation. ${ }^{2,5}$ CAIs have been utilized in treatment of macular edema in a number of ocular disorders, and the addition of a CAI to a strong steroid and NSAID can help advance treatment, particularly when other topical regimens have failed and more invasive therapy is being considered. IRB approval was deemed not applicable for this case series. Patients in this review provided signed voluntary and informed consent to the described treatment and for publication of their results. Patients in this review displayed appropriate capacity to provide consent. Patients understood the risks, benefits, and alternatives for the triple therapy treatment and understood they were entitled to withdraw previous consent at any time during the treatment.

\section{Case reports}

\section{Cases I-3: Irvine-Gass syndrome} Case I

A 70-year-old female noted progressive vision loss 2 weeks following uncomplicated cataract surgery in the right eye. Best-corrected visual acuity (BCVA) measured 20/100, intraocular pressure (IOP) was $16 \mathrm{mmHg}$, and fluorescein angiography (FA) studies revealed CME with disc leakage (Figure 1A). Optical coherence tomography (OCT) studies revealed cystic edema with a central macular thickness (CMT) of $424 \mu \mathrm{m}$ (Figure 1A). She had been treated with prednisolone acetate three times a day
(TID). We switched the patient to difluprednate $0.05 \%$, nepafenac $0.1 \%$, and brinzolamide $1 \%$, all TID, in the right eye. After 13 days, BCVA measured 20/20 with an IOP of $15 \mathrm{mmHg}$. CMT decreased $97 \mu \mathrm{m}$ (Figure 1B). We slowly tapered off the difluprednate and brinzolamide over a period of 7 months given the recurrence noted after discontinuing the drops. She is now maintained on nepafenac twice daily (BID) with a BCVA of 20/20 and CMT of $293 \mu \mathrm{m}$ (Figure 1C).

\section{Case 2}

A 75-year-old female with a history of epiretinal membrane (ERM) removal approximately 1 year prior to presentation to the office noted progressive vision loss 5 months following uncomplicated cataract surgery in the right eye. BCVA measured 20/40-2 and IOP was $7 \mathrm{mmHg}$. FA studies showed CME with disc leakage (Figure 2A) and OCT studies revealed cystic edema with a CMT of $461 \mu \mathrm{m}$ (Figure 2A). She was started on bromfenac $0.07 \%$ once daily (QD) for 9 months prior to her presenting visit given her history of ERM. Given the increased edema on bromfenac alone, we switched her to difluprednate, nepafenac, and brinzolamide TID in the right eye. After 14 days, BCVA measured 20/25 with a CMT of $294 \mu \mathrm{m}$, a $167 \mu \mathrm{m}$ improvement (Figure 2B), and her IOP was controlled at $13 \mathrm{mmHg}$. The difluprednate was tapered weekly and stopped after 4 weeks, and the nepafenac and brinzolamide were continued BID. At week 6, however, her

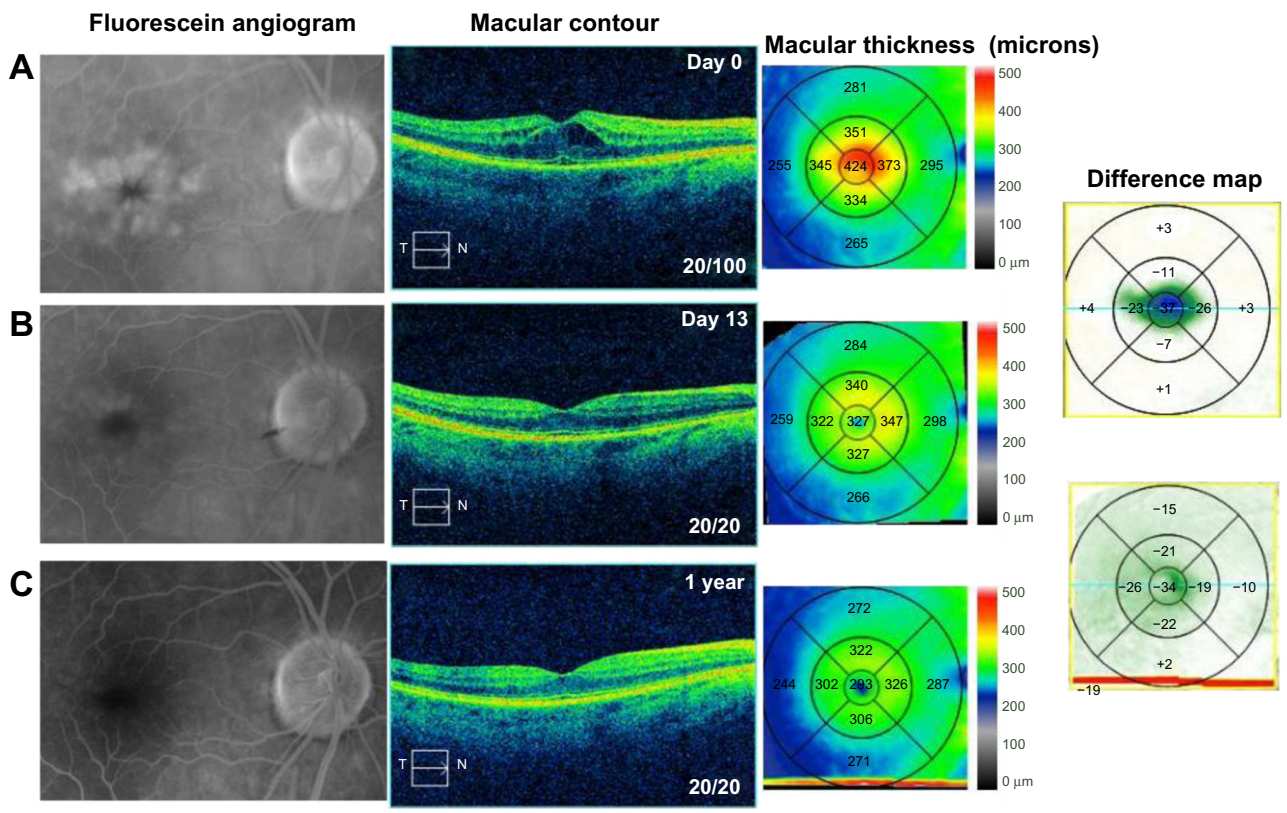

Figure I Fluorescein angiography and optical coherence tomography studies for case I (Irvine-Gass syndrome).

Notes: (A) Cystoid macular edema, macular contour, and macular thickness at presentation. (B and $\mathbf{C})$ Reduced macular leakage on the fluorescein angiogram; reduced macular contour and thickness; and macular change analysis (B) 13 days following treatment and (C) I year following treatment. The difference maps are made between the intervals between the figures. The first difference map shows the difference between day 13 and day 0 . The second difference map shows the difference between I year and day 13 . Abbreviations: $\mathrm{T}$, temporal; $\mathrm{N}$, nasal. 

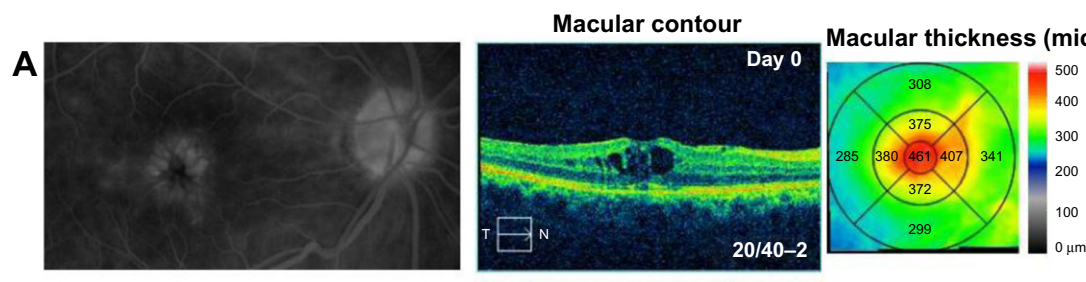

$\mathbf{B}$
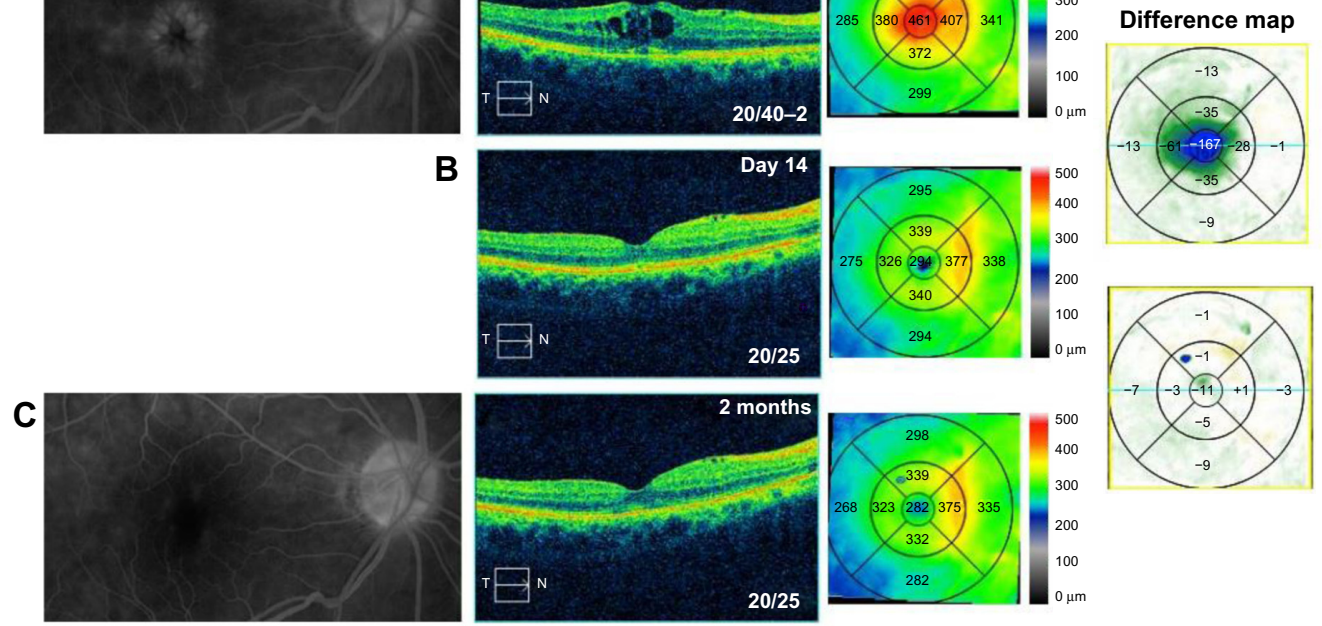

Figure 2 Fluorescein angiography and optical coherence tomography studies for case 2 (Irvine-Gass syndrome).

Notes: (A) Cystoid macular edema, macular contour, and macular thickness at presentation. (B) Macular contour and thickness and macular change analysis 14 days following treatment. (C) Decreased macular leakage; decreased macular contour and thickness; and macular change analysis 2 months following treatment.

Abbreviations: $\mathrm{T}$, temporal; $\mathrm{N}$, nasal.

vision decreased to 20/30 and the CME had recurred. She was once again started on a tapering course of difluprednate and asked to continue nepafenac and brinzolamide TID. After 3 additional months of continued treatment, her BCVA improved to $20 / 25$ with CMT of $282 \mu \mathrm{m}$ and reduction in CME (Figure 2C). She continues to be maintained on nepafenac and dorzolamide-timolol 2\%/0.5\% BID given her history of ERM removal. The highest measured IOP was $18 \mathrm{mmHg}$ and no additional glaucoma medications were added.

\section{Case 3}

An 81-year-old male noted painless progressive decreased vision 2 weeks following uncomplicated cataract surgery in the right eye. BCVA measured 20/40-2 and IOP was $13 \mathrm{mmHg}$. OCT studies revealed cystic edema associated with a mild ERM and CMT of $375 \mu \mathrm{m}$ (Figure $3 \mathrm{~A}$ ). He was started on difluprednate, nepafenac, and brinzolamide TID in the right eye. After 14 days, BCVA measured 20/25 with CMT of $302 \mu \mathrm{m}$, a $73 \mu \mathrm{m}$ improvement (Figure 3B), and IOP was unchanged at $13 \mathrm{mmHg}$. He continued to improve
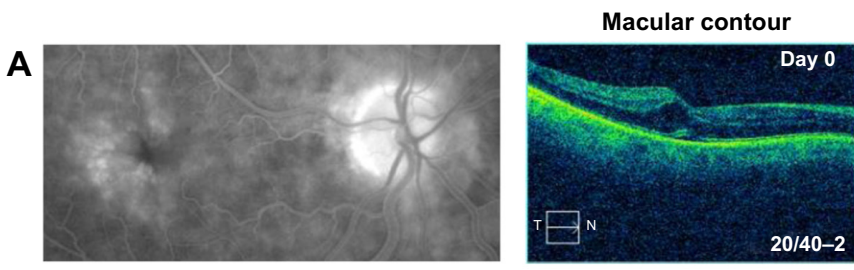

Macular thickness (microns)

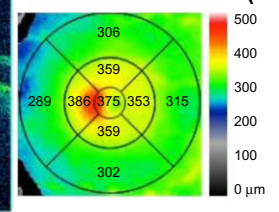

B
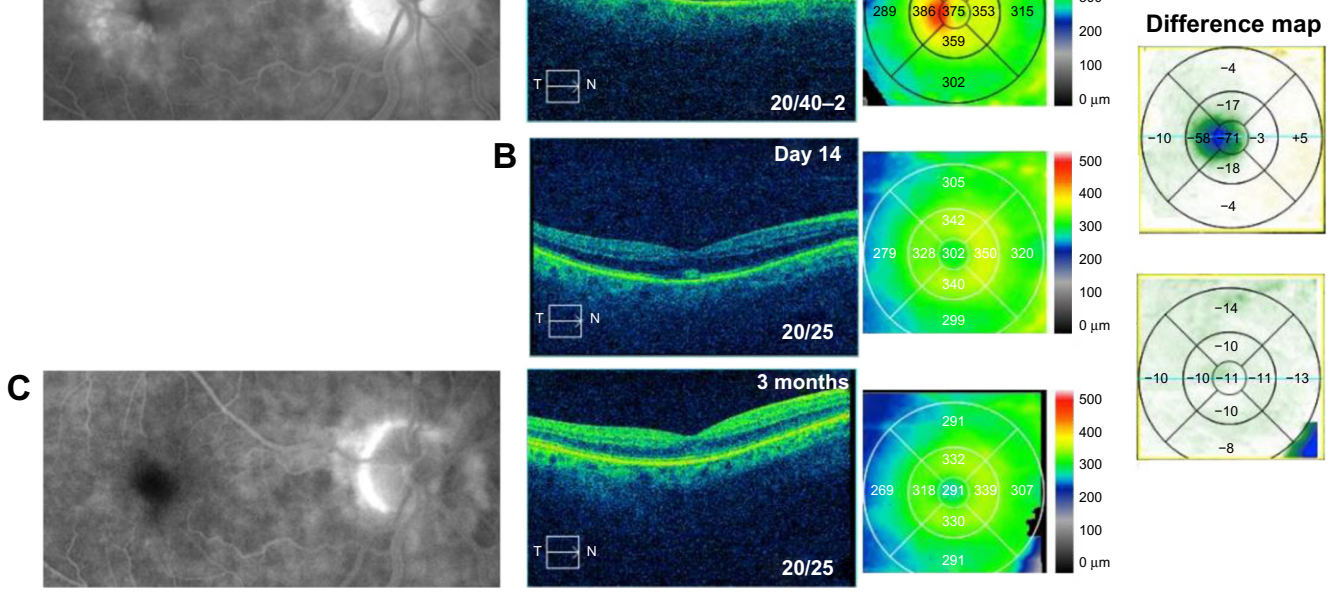

Figure 3 Fluorescein angiography and optical coherence tomography studies for case 3 (Irvine-Gass syndrome).

Notes: (A) Cystoid macular edema, macular contour, and macular thickness at presentation. (B) Macular contour and thickness and macular change analysis 14 days following treatment. (C) Decreased macular leakage; decreased macular contour and thickness; and macular change analysis 3 months following treatment. Abbreviations: $\mathrm{T}$, temporal; $\mathrm{N}$, nasal. 
and the frequency of his drops was decreased to QD. After 4 months of treatment, his BCVA stabilized at 20/25 and CMT measured $291 \mu \mathrm{m}$ with a further $11 \mu \mathrm{m}$ decrease in CMT (Figure 3C). The difluprednate was then discontinued and he was maintained on ketorolac $0.5 \%$ and dorzolamide $2 \%$ QD. He developed iatrogenic keratopathy after 9 months, so all drops were discontinued and he received a subtenon triamcinolone acetonide $40 \mathrm{mg} / \mathrm{mL}$ injection in the right eye to treat the mild residual CME. This further improved his vision to 20/20 10 months following cataract surgery.

\section{Case 4: diabetic retinopathy}

A 62-year-old male noted progressive decreased vision in the left eye and presented with CME secondary to diabetic microaneurysms with associated exudates. BCVA measured 20/25-2, IOP was $12 \mathrm{mmHg}$, and OCT studies showed CME with a CMT of $284 \mu \mathrm{m}$ (Figure 4A). He was started on difluprednate and brinzolamide TID in the left eye, as well as bromfenac QD in the left eye. After 14 days, BCVA remained 20/25-2, but his CMT had minimally improved by $9 \mu \mathrm{m}$ and a reduction in the perifoveal cyst was noted (Figure 4B). The macular exudates resolved as noted on clinical exam. He underwent cycles of initiation and cessation of treatment over a 10-month period. There was consistent recurrence of CME after cessation of drops (Figure 4C) and improvement when the topical combination of drops was reinitiated (Figure 4D). Following this 10-month period, he was tapered off difluprednate and brinzolamide, then switched to ketorolac for

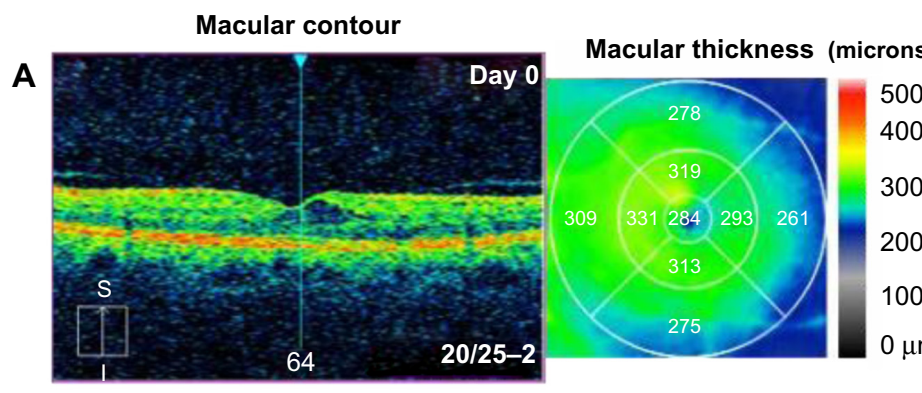

в

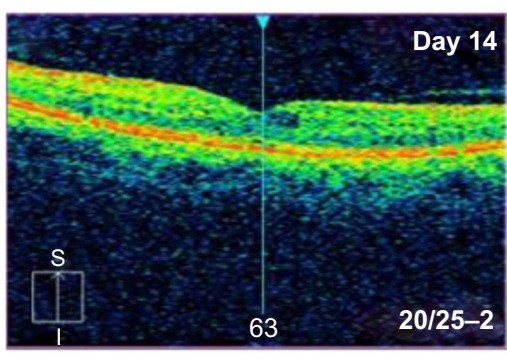

c

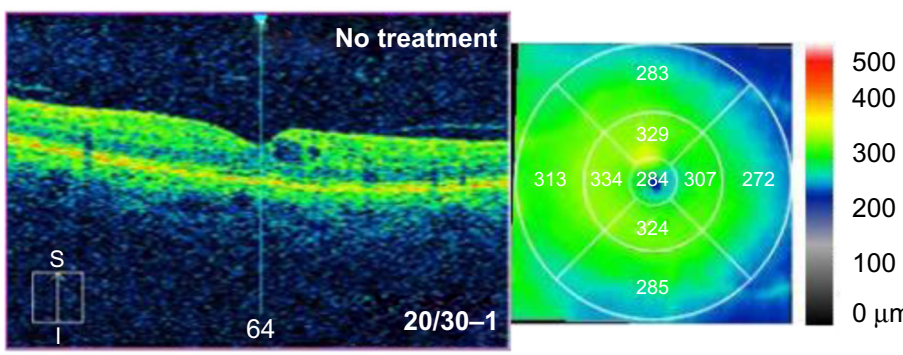

D

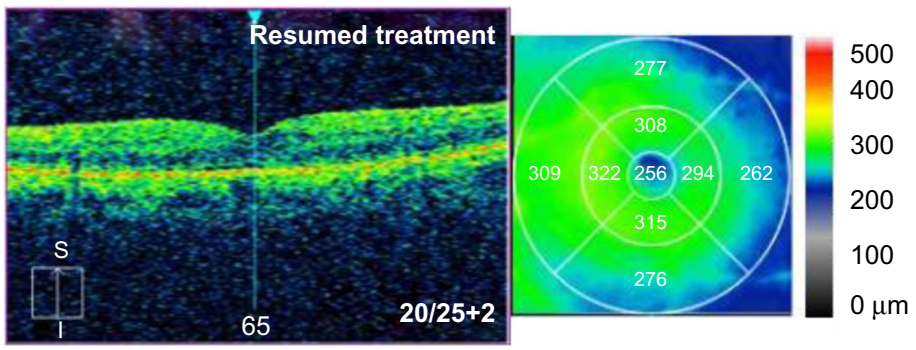

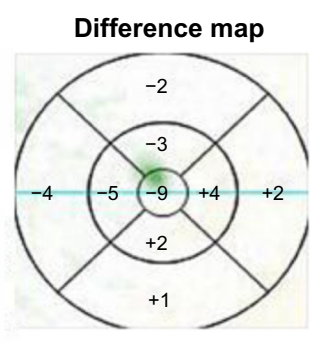
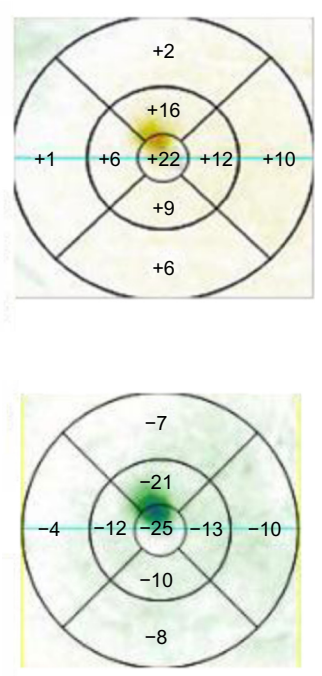

Figure 4 Optical coherence tomography studies for case 4 (diabetic retinopathy).

Notes: (A) Macular contour and thickness at presentation. (B) Macular contour and thickness and macular change analysis I4 days following treatment. (C) Increased cystoid macular edema with cessation of treatment and (D) improvement with resumption of treatment.

Abbreviations: S, superior; I, inferior. 
coverage reasons, and has been 20/20 in the left eye for the past year on ketorolac BID.

\section{Case 5: uveitis}

A 73-year-old pseudophakic female noted severe progressive vision loss in both eyes, the left eye greater than the right eye, and was diagnosed with CME secondary to birdshot chorioretinopathy with panuveitis and a positive HLA-A29. BCVA measured 20/50+2 in the right eye and 20/200 in the left eye with IOP of $12 \mathrm{mmHg}$ in the right eye and $13 \mathrm{mmHg}$ in the left eye. OCT studies revealed CME in both eyes with CMT of $343 \mu \mathrm{m}$ in the right eye and $372 \mu \mathrm{m}$ in the left eye (Figure 5). Clinical exam revealed $1+$ cells in the anterior chamber and $3+$ in the vitreous in both eyes. CME was also confirmed with FA studies. She was started on difluprednate four times a day and dorzolamide-timolol and nepafenac BID in both eyes. After 2 weeks, BCVA was 20/40-2 in the right eye and 20/80-1 in the left eye, and CMT had decreased by $82 \mu \mathrm{m}$ in the right eye and $93 \mu \mathrm{m}$ in the left eye (Figure 5). IOP measured $12 \mathrm{mmHg}$ in the right eye and $10 \mathrm{mmHg}$ in the left eye. She has been maintained on difluprednate TID, dorzolamide-timolol TID, and nepafenac BID, all in both eyes, for the past 2 months; her BCVA remains stable, and her edema continues to improve.

\section{Case 6: branch retinal vein occlusion}

A 53-year-old male noted progressive pericentral blurring in the right eye for 4 days associated with pain in the right eye. Uncorrected visual acuity (UCVA) measured 20/25 in the right eye and 20/20 in the left eye with an IOP of $11 \mathrm{mmHg}$ in both eyes. Fundoscopic exam revealed macular edema and retinal hemorrhages associated with a branch retinal vein occlusion in the right eye. OCT studies revealed CME with CMT of $356 \mu \mathrm{m}$ (Figure 6A). FA studies were performed and confirmed branch retinal vein occlusion with CME. He was started on difluprednate, nepafenac, and brinzolamide, all TID, in the right eye. After 7 days, the patient reported an increase in vision with decreasing blurriness. UCVA remained 20/25 in the right eye and 20/20 in the left eye with IOP of $12 \mathrm{mmHg}$ in the right eye and $10 \mathrm{mmHg}$ in the left eye. OCT studies showed a $49 \mu \mathrm{m}$ reduction in CMT and $74 \mu \mathrm{m}$ reduction in perifoveal thickness with near resolution of the CME (Figure 6B). He continued brinzolamide and nepafenac, while slowly tapering off of the difluprednate (BID for 1 week, QD for 1 week, thrice weekly for 1 week, twice weekly for 1 week, then discontinue). UCVA ultimately recovered to $20 / 20$ in both eyes with IOP of $16 \mathrm{mmHg}$ in the right eye and $14 \mathrm{mmHg}$ in the left eye 3 months following treatment (Figure 6C). At that time, the patient was deemed stable and nepafenac and brinzolamide were discontinued.

The patient was lost to follow-up for 1 year and returned noting progressive blurring in the right eye. UCVA measured 20/30 in the right eye and 20/25 in the left eye. IOP measured $14 \mathrm{mmHg}$ in the right eye and $13 \mathrm{mmHg}$ in the left eye. OCT study showed recurrent CME with a $44 \mu \mathrm{m}$ increase in CMT and $82 \mu \mathrm{m}$ increase perifoveally (Figure 6D). The patient was started on bromfenac $0.07 \%$ QD and brinzolamide/brimonidine $1 \% / 2 \%$ BID in the right eye, as well as difluprednate BID in the left eye. One month following treatment, the patient recovered to $20 / 20-1$ in the right eye and 20/20 in the left eye (Figure 6E). IOP was elevated at $24 \mathrm{mmHg}$ in the right eye and $18 \mathrm{mmHg}$ in the left eye, so the brinzolamide/brimonidine was increased to TID in the right eye and the difluprednate was reduced to QD in the left eye.

\section{Discussion}

Among the several etiologies of CME in macular disease, they all have the common underlying mechanism of inflammation. Prostaglandins (PGs) are the classic inflammatory mediators generated through the arachidonic acid cascade. They act directly to increase vascular permeability and cause leakage by binding PG receptors on capillary endothelial cells and
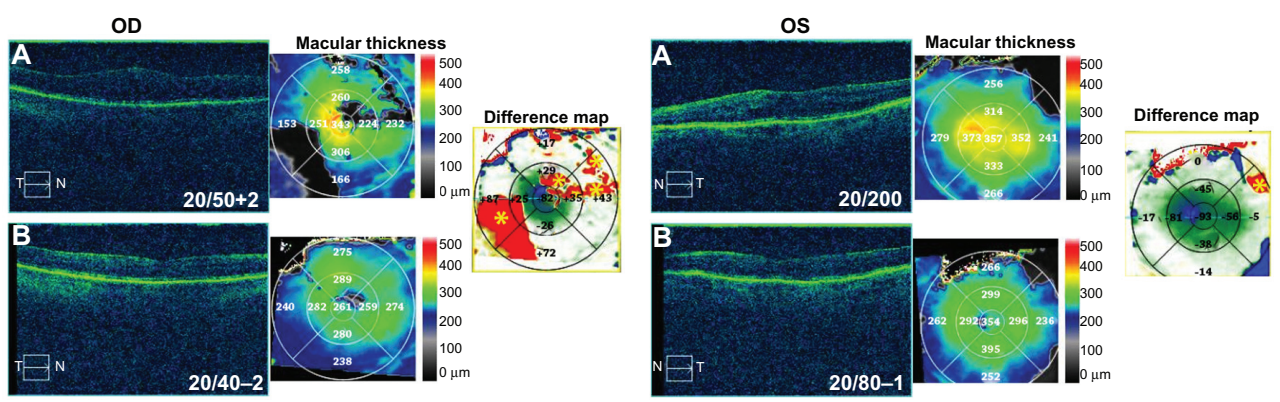

Figure 5 Optical coherence tomography studies for case 5 (uveitis).

Notes: (A) Macular contour and thickness at presentation. (B) Macular contour, thickness, and macular change analysis (difference map) following treatment. Yellow asterisks indicate artifacts.

Abbreviations: $\mathrm{T}$, temporal; $\mathrm{N}$, nasal; OD, oculus dexter; OS, oculus sinister. 
A
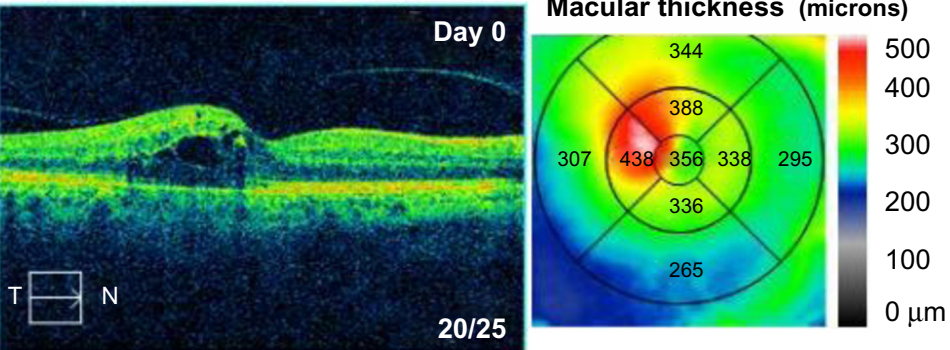

B
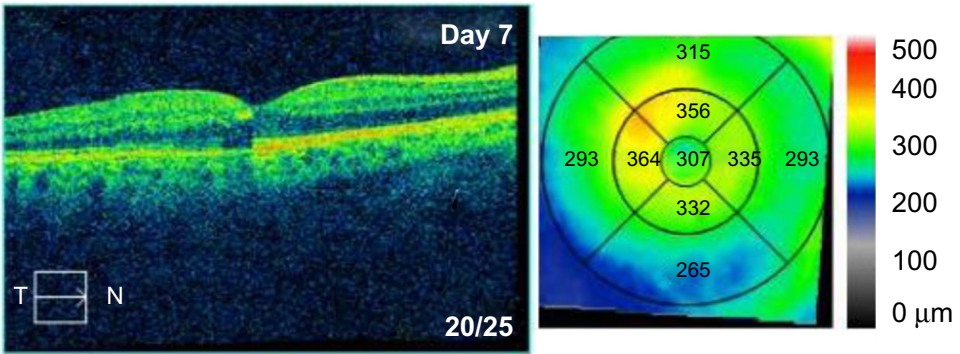

C
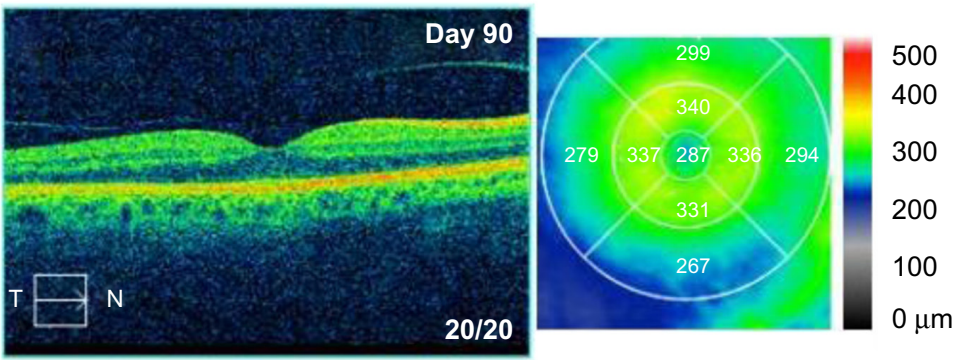

D
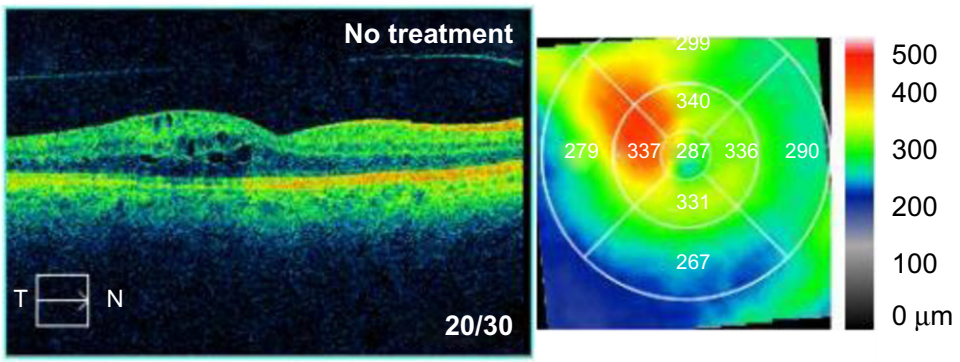

E

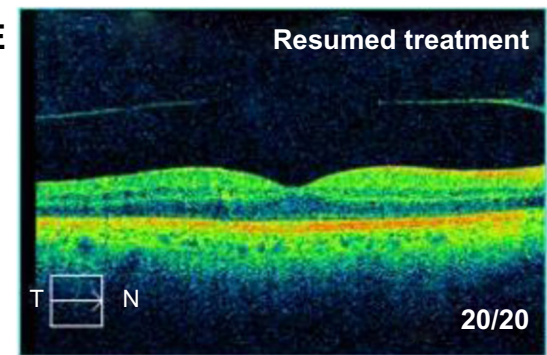

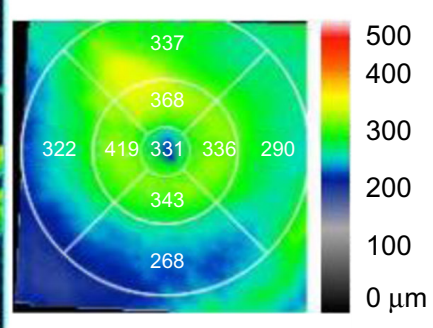

\section{Difference map}
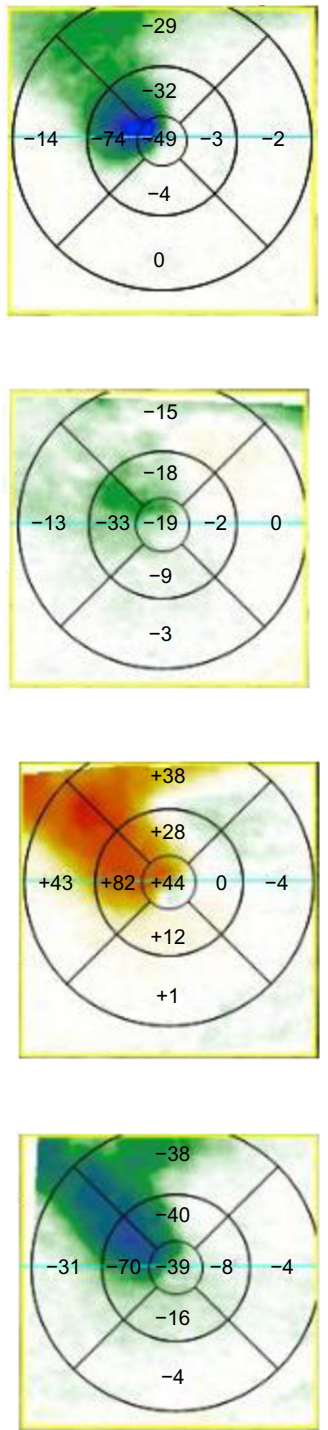

Figure 6 Optical coherence tomography studies for case 6 (branch retinal vein occlusion).

Notes: (A) Macular contour and thickness at presentation. (B and $\mathbf{C})$ Macular contour and thickness and macular change analysis (B) 7 days and (C) 90 days following treatment. (D) Increased cystoid macular edema with cessation of treatment and (E) improvement with resumption of treatment.

Abbreviations: T, temporal; N, nasal.

facilitating the opening of the tight junctions between them, and indirectly by upregulation of vascular endothelial growth factor (VEGF) and carbonic anhydrase-1 (CA-1) expression and downregulation of $\mathrm{K}+$ channels in Müller cells. ${ }^{6}$ This causes cystic edema by increasing the influx and reducing the efflux of ions and fluid from the inner nuclear layer and Henle fiber layer in the macular region. ${ }^{7}$

Currently, topical nonsteroidal anti-inflammatory drops and topical corticosteroids are used as the primary form of management of CME, as these agents are directed at 
decreasing intraocular PG levels, which are implicated in the pathogenesis of CME as described above. ${ }^{3}$ Oral acetazolamide, a CAI, has been utilized in select cases where topical treatment has failed. ${ }^{7}$ Intravitreal and subtenon injections of corticosteroids have also been utilized in the treatment of CME; however, their use remains limited by side effects, duration of effect, and risks associated with the procedure itself. Heier et al demonstrated in their study that treatment of acute CME was best accomplished with combination therapy of corticosteroids and NSAIDs, and additional previous works have demonstrated this synergism. ${ }^{2}$

The present study evaluated the efficacy of a combination cocktail of drops, the "strong" steroid difluprednate, an NSAID, and a CAI, all of which have been used as monotherapy in the management of CME. ${ }^{4}$ This combination of medications works not only to reduce the levels of PG synthesized within the affected eye, but also to reverse the associated hyperpermeability of the blood-retinal barrier.

Difluprednate ophthalmic emulsion $0.05 \%$ is a very potent ("strong") corticosteroid, which inhibits the enzyme phospholipase A2, lowering the amount of arachidonic acid available to COX-1 and -2 for conversion to PGs. It has greater potency than prednisolone acetate, and its formulation as an emulsion allows for better dose uniformity when compared to prednisolone..$^{5}$ This can account for the equal efficacy of difluprednate four times a day to prednisolone acetate every 2 hours in the treatment of anterior uveitis and associated macular edema. ${ }^{8}$ Topical NSAIDs synergize with steroids in the treatment of CME by inhibiting the activity of COX-1 and COX-2. ${ }^{9}$

CAI drops alone have long been known to be effective for treatment of macular edema in several ocular conditions. ${ }^{10}$ They have been shown to reduce edema in patients with choroideremia, ${ }^{11}$ and more recently were included in a treatment regimen for Vogt-Koyanagi-Harada disease. ${ }^{12}$ CAIs induce acidification of the subretinal space, which is responsible for increasing fluid resorption from the retina through the retinal pigment epithelium (RPE) in the choroid plexus. ${ }^{10} \mathrm{CAIs}$ also increase fluid transport across the human RPE, thus reducing edema, ${ }^{13,14}$ and it is hypothesized that the site of action for CAIs in the treatment of CME is the RPE rather than fluid movement across retinal vessels. ${ }^{10}$ Carbonic anhydrase is also an inflammatory mediator, so a secondary benefit may be suppression of the inflammatory process underlying the vascular and RPE leakage causing the CME.

Topical CAIs were also selected for the cocktail to counter IOP spikes, which can occur with topical steroids such as difluprednate. ${ }^{5}$ Glucocorticoids have long been associated with elevated IOP, with $30 \%-40 \%$ of the general population showing a measurable increase in IOP. In case 2 , the patient experienced an IOP increase from $7 \mathrm{mmHg}$ to $18 \mathrm{mmHg}$, an increase of $11 \mathrm{mmHg}$, over a 4-month period of treatment. The IOP increase was likely associated with the pulses of high-dose steroids, but was managed and remained stable with reduction of the strong steroid and use of the CAI.

Caution is advised with the long-term use of topical medications as they can exacerbate or create corneal surface disease. ${ }^{15}$ Toxic responses often occur because of the preservatives or excipients in the formulations, rather than the drugs themselves. ${ }^{15}$ In case 3 , the patient developed an iatrogenic keratopathy due to long-term use of topical medications. Invasive therapy was thus initiated for this patient in order to continue management of the CME. This illustrates one caveat in using combination topical therapy, and further studies could be undertaken to more clearly delineate the side effect profile of this treatment modality; however, it does not detract from the efficacy of the combination therapy, as the patient's BCVA did improve from 20/40 to 20/25 in the right eye prior to his developing a keratopathy.

There are some limitations to our case series, the first being lack of an appropriate control group. We did not want to withhold treatment in any of our patients, as untreated CME can lead to permanent deficits in visual acuity, thus a control arm was not utilized. While we did note a dramatic improvement in CME in patients undergoing treatment with other medications prior to addition of the cocktail, spontaneous resolution of CME has been reported in previous studies, and it is impossible to state with certainty that a combination treatment is efficacious without a placebo-controlled arm and without a standardized dosing regimen. ${ }^{2}$ Future studies could address this weakness by comparing our combination treatment to either placebo or to the standard of care. The second weakness of our study is the small sample size. Although we only present six cases in this review, clinical experience has clearly demonstrated the efficacy of triple therapy compared to monotherapy and observation. While the relative contribution of each agent to the treatment of CME is not defined in this study, clinical experience and prior studies suggest that the effects would be additive. ${ }^{2}$

Controlled and randomized studies comparing this combination against placebo, topical monotherapy, and more invasive treatments such as subtenon or intravitreal steroid injection should be undertaken to determine the efficacy of this triple-therapy combination over other treatment options. 


\section{Conclusion}

The topical cocktail of "strong" steroid (difluprednate), NSAID, and CAI drops is efficacious against complex cases of CME. The individual elements may have a synergistic effect on decreasing CME, and this cocktail may even rival an intravitreal injection over time (Gallemore, unpublished data 2014). We recommend this topical cocktail as a noninvasive local therapy for more severe cases of severe CME, particularly when other topical regimens have failed.

\section{Disclosure}

The authors report no conflicts of interest in this work.

\section{References}

1. Lardenoye CW, van Kooij B, Rothova A. Impact of macular edema on visual acuity in uveitis. Ophthalmology. 2006;113:1446-1449.

2. Heier JS, Topping TM, Baumann W, Dirks MS, Chern S. Ketorolac versus prednisolone versus combination therapy in the treatment of acute pseudophakic cystoid macular edema. Ophthalmology. 2000;107(11): 2034-2038.

3. Ray S, D'Amico DJ. Pseudophakic cystoid macular edema. Semin Ophthalmol. 2002;17:167-180.

4. Ober MD, Klais CM, Cunningham ET Jr. Management Options for Macular Edema: Many strategies have been used to manage macular edema, with varying degrees of success [webpage on the Internet]. Newtown Square: Review of Ophthalmology; 2005. Available from: http://www.reviewofophthalmology.com/content/d/retinal_insider/ i/1310/c/25220/. Accessed July 15, 2015.
5. Mithal C, Singh S, Gupta S, Mithal S. Difluprednate: an overview. Del J Ophthalmol. 2013;23(3):165-168.

6. Vinores SA, Sen H, Campochiaro PA. An adenosine agonist and prostaglandin E1 cause breakdown of the blood-retinal barrier by opening tight junctions between vascular endothelial cells. Invest Ophthalmol Vis Sci. 1992;33:1870-1878.

7. Bringmann A, Reichenbach A, Wiedemann P. Pathomechanisms of cystoid macular edema. Ophthalmic Res. 2004;36:241-249.

8. Jamal KN, Callanan DG. The role of difluprednate ophthalmic emulsion in clinical practice. Clin Ophthalmol. 2009;3:381-390.

9. Gallemore RP. NSAIDs in treatment of retinal disorders. Review of Ophthalmology. 2006;81-89.

10. Wolfensberger TJ. The role of carbonic anhydrase inhibitors in the management of macular edema. Doc Ophthalmol. 1999;97(3-4): 387-397.

11. Genead MA, McAnany JJ, Fishman GA. Topical dorzolamide for treatment of cystoid macular edema in patients with choroideremia. Retina. 2012;32(4):826-833.

12. Onishi SM, Asahi MG, Chou C, Gallemore RP. Topical difluprednate for the treatment of Harada's disease. Clin Ophthalmol. 2015;9:157-167.

13. Gallemore RP, Hughes BA, Miller SS. Retinal pigment epithelial transport mechanisms and their contributions to the electroretinogram. Prog Retin Eye Res. 1997;16:509-566.

14. Cox SN, Hay E, Bird AC. Treatment of chronic macular edema with acetazolamide. Arch Ophthalmol. 1988;106:1190-1195.

15. Dart J. Corneal toxicity: the epithelium and stroma in iatrogenic and factitious disease. Eye (Lond). 2003;17;886-892.
International Medical Case Reports Journal

\section{Publish your work in this journal}

The International Medical Case Reports Journal is an international, peer-reviewed open-access journal publishing original case reports from all medical specialties. Previously unpublished medical posters are also accepted relating to any area of clinical or preclinical science. Submissions should not normally exceed 2,000 words or

\section{Dovepress}

4 published pages including figures, diagrams and references. The manuscript management system is completely online and includes a very quick and fair peer-review system, which is all easy to use. Visit http://www.dovepress.com/testimonials.php to read real quotes from published authors. 